\title{
Three-tube method and covered metallic stent for the treatment of anastomotic leakage after esophagectomy
}

\author{
Yonghua $\mathrm{Bi}^{\dagger}$, Zhengyang $\mathrm{Wu}^{\dagger}$, Mengfei Yi, Xinwei Han ${ }^{*}$ [D and Jianzhuang Ren ${ }^{*}$
}

\begin{abstract}
Background: Anastomotic leakage is common and life-threatening complication after esophagectomy. The management of esophageal anastomotic leakage remains challenging. We aimed to determine the safety, feasibility and efficacy of three-tube method and covered metallic stent placement for the management of anastomotic leakage.

Methods: Twenty-six consecutive patients with anastomotic leakage were treated using three-tube method and covered metallic stent and the medical records were retrospectively assessed. All patients received placement of abscess drainage tube, jejunal feeding tube and gastrointestinal decompression tube as well as esophageal covered stent, followed by continue abscess drainage, nutritional support and anti-inflammatory treatment. Tubes and esophageal stents will be removed once anastomotic leakage heals.

Results: The procedure was technically successful in 23 patients (95.8\%). A total of 31 covered stents were used. Esophageal stents and abscess drainage tubes were successfully removed from 14 patients. The median retention duration was 2.3 months and 2.6 months for stent and abscess drainage tubes, respectively. No perioperative death, esophageal rupture, massive hemorrhage, or other severe complications were observed during procedures. The abscess cavity had markedly decreased in 8 patients or disappeared in 16 cases. During follow-up, 8 patients died of cancer recurrence and 2 patients died of severe pulmonary infection. The 1-, 3-, 5-year survival rates were 60.1, 51.5 and $51.5 \%$, respectively.
\end{abstract}

Conclusion: Three-tube method and covered metallic stent placement is safe, feasible and efficacious for treatment of anastomotic leakage after esophagectomy.

Keywords: Anastomotic leakage, Esophageal stent, Drainage, Three-tube method

\section{Background}

Anastomotic leakage is a rare but life-threatening complication of esophagectomy for esophageal cancer or esophagogastric carcinoma [1,2], with an overall mortality rate of 20 to $50 \%$ [3-6]. Contamination in the abscess cavity may corrode vessels and even result in a higher

\footnotetext{
* Correspondence: dreamweaver08@126.com; rrjjzzjrk@126.com $\mathrm{BYH}$ and WZY share co-first author.

${ }^{\dagger}$ Yonghua Bi and Zhengyang Wu contributed equally to this work. Department of Interventional Radiology, The First Affiliated Hospital of Zhengzhou University, No.1, East Jian She Road, Zhengzhou 450052, China
}

mortality [7]. Various conservative treatment protocols have been used for the management of anastomotic leakage over the past two decades, including the application of biodegradable fistulae plugs or fibrin glue, endoscopic transluminal drainage or clipping and metallic esophageal stent insertion [4, 8-10]. Despite these modalities, management of anastomotic leakage remains challenging and the optimal treatment protocol need to be determined $[2,4,11]$.

C C The Author(s). 2020 Open Access This article is licensed under a Creative Commons Attribution 4.0 International License, which permits use, sharing, adaptation, distribution and reproduction in any medium or format, as long as you give appropriate credit to the original author(s) and the source, provide a link to the Creative Commons licence, and indicate if changes were made. The images or other third party material in this article are included in the article's Creative Commons licence, unless indicated otherwise in a credit line to the material. If material is not included in the article's Creative Commons licence and your intended use is not permitted by statutory regulation or exceeds the permitted use, you will need to obtain permission directly from the copyright holder. To view a copy of this licence, visit http://creativecommons.org/licenses/by/4.0/ The Creative Commons Public Domain Dedication waiver (http://creativecommons.org/publicdomain/zero/1.0/) applies to the data made available in this article, unless otherwise stated in a credit line to the data. 
In this study, three-tube method (abscess drainage tube, jejunal feeding tube and gastrointestinal decompression tube) and esophageal covered stent was used. We aimed to determine the safety, feasibility and efficacy of this protocol for the management of anastomotic leakage.

\section{Methods}

\section{Patient selection}

This study was approved by the Ethics Committee Board of Zhengzhou University First Affiliated Hospital. Informed consent was obtained from all patients. This study enrolled all patients with anastomotic leakage after esophagectomy who received three-tube method and esophageal covered stent placement in our institution between April 2011 and July 2018. The diagnosis of an anastomotic leakage was made based on esophagography (Fig. 1a-b) and chest computed tomographic scan (Fig. 1c-d). During the observation period, there were no changes in technique. Three-tube method was used due to financial difficulties or the position of the fistula was not suitable for stent placement. Patients were divided into 2 groups dependent on the size of the leak: small leaks (less than $6 \mathrm{~mm}$ ) and large leaks (more than $6 \mathrm{~mm})$.

\section{Three-tube method}

All interventional procedures were performed under fluoroscopic guidance, local anesthesia and conscious sedation. The esophagus and pharyngonasal cavity was anesthetized with an oral lidocaine gel and a tetracaine spray, respectively. A 5-F cobra catheter was introduced through the outlet of anastomotic leakage and into the abscess cavity. The catheter tip was inserted into the distal end of the abscess cavity, followed by exchange with a 5-F pigtail or straight catheter (Cook Medical, Inc., Bloomington, IN). Continuous negative pressure suction was performed using a $20 \mathrm{ml}$ syringe thereafter, with an appropriate pressure to drain the abscess at the same time avoiding excessive negative pressure in order not to cause iatrogenic injury and bleeding. We reduced negative pressure or stopped aspirating in case of bleeding. The abscess cavity was repeatedly rinsed with 100-200 $\mathrm{ml}$ of saline. A jejunal feeding tube and gastrointestinal
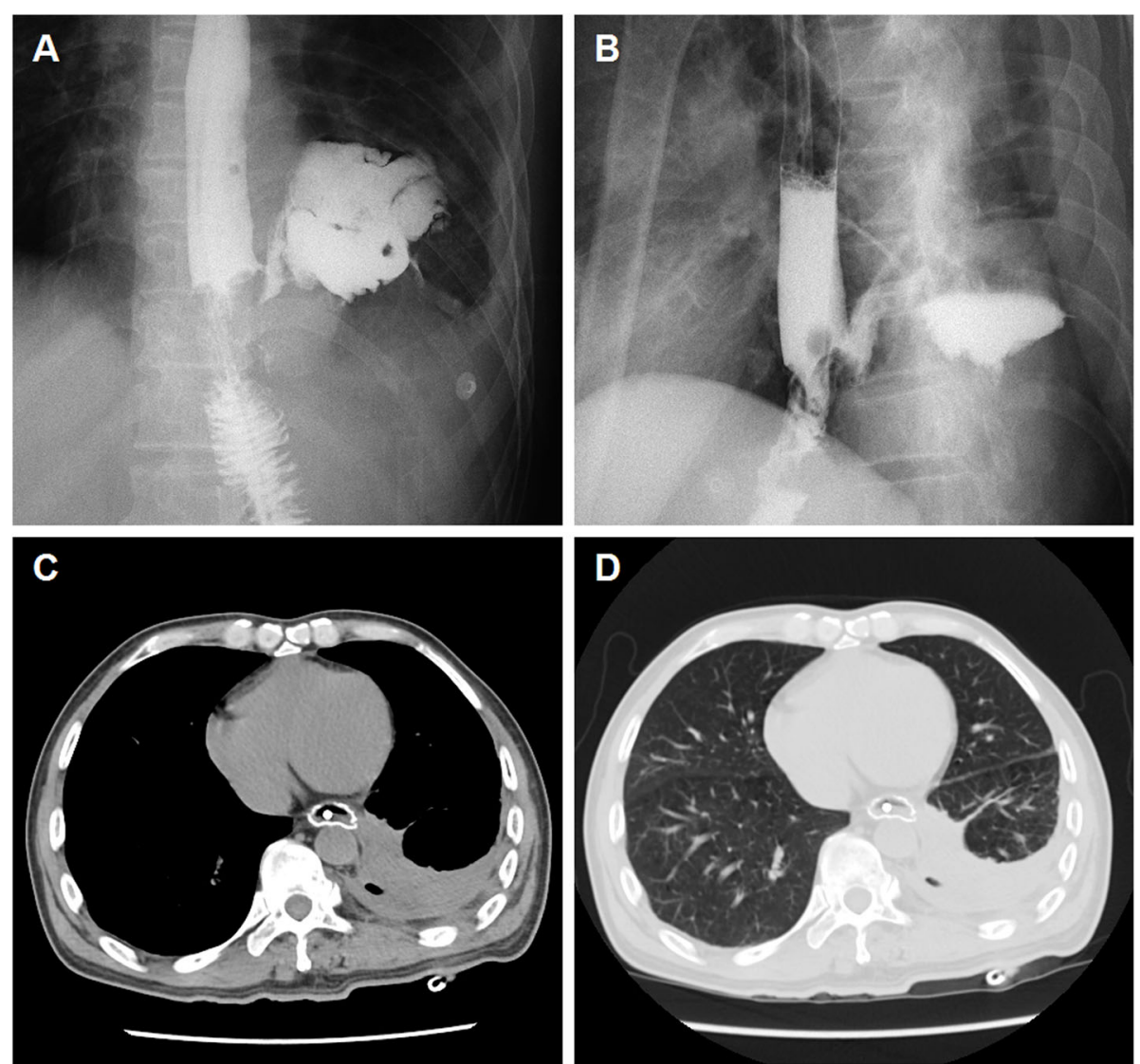

Fig. 1 A 59-year-old man with anastomotic leak after esophagojejunostomy (case 24). a-b Esophagography showing an anastomotic leakage in the lower esophagus and irregular abscess in the pleural cavity. $\mathbf{c}-\mathbf{d}$ Chest CT scan in the mediastinal and lung windows show mediastinal abscess and a small amount of pleural effusion before procedure 
decompression tube was inserted into the jejunum and gastric cavity, respectively. Enteral nutrition solution was infused via the jejunal feeding tube. Patients were allowed to resume oral intake once the leaks had been sealed by the covered stent and confirmed by contrast study. Broad-spectrum antibiotic treatment was performed before and after procedure.

\section{Esophageal covered stent placement}

All patients received fluoroscopic placement of esophageal covered stent (Nanjing Micro-Tech Medical Company, Nanjing, China). The stent diameter ranged from 18 to $22 \mathrm{~mm}$ and stent length ranges from 70 to $160 \mathrm{~mm}$. A 5-F catheter was inserted transorally into the gastric cavity and then a stiff guide wire was introduced. A covered stent was delivered via the stiff guide wire and then released carefully to block the leakage. A stent fixation line was used for fixation and stent adjustment. Leakage closure was confirmed by repeated esophagography (Fig. 2a-b). About 5 to 7 days after stent placement, chest CT and esophagography were performed again to study whether esophageal stent fit watertight, as well as the change of the abscess cavity and the position of abscess drainage tube (Fig. 2c-d). Stent adjustment or second stent placement were performed if esophageal stent does not fit watertight. The tube was adjusted to make sure effective drainage if necessary. The drainage tubes and stents were removed if complete disappearance of abscess cavity and full expansion of the lungs was confirmed by esophagography (Fig. 3a-b) and chest CT showed (Fig. 3c-d).

\section{Results}

\section{General information}

This study involved a total of 24 patients with anastomotic leakage, including 21 men and 3 women (Table 1). The age range of patients was 43 years to 76 years, with a median age of 59.5 years. Half of patients showed normal temperature after esophagectomy, the remained patients showed fever, with a median temperature of
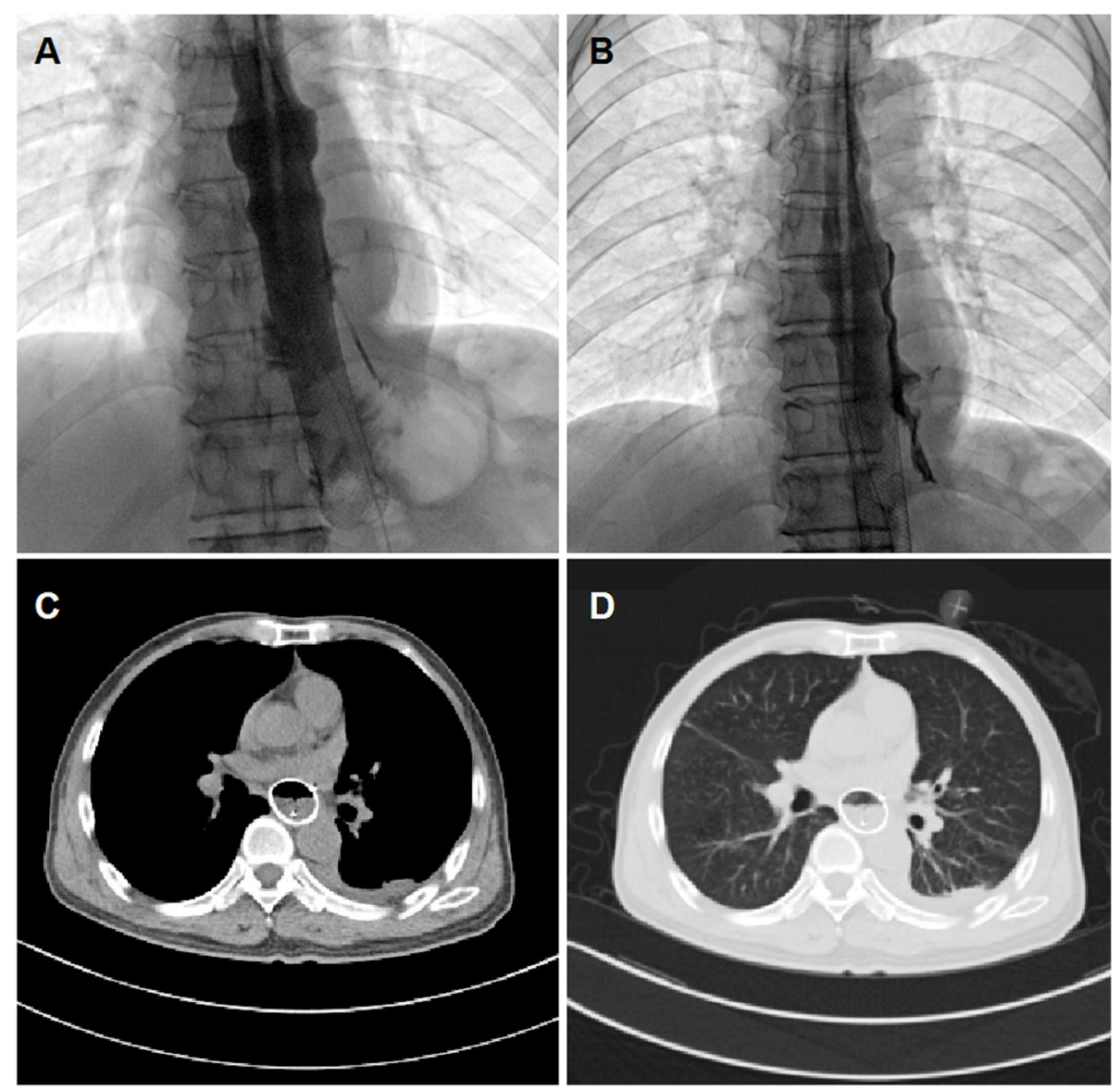

Fig. 2 Three-tube method and covered stent placement for case 24. a After esophageal covered stent and drainage tube placement, esophagography shows that the contrast agent flows though the esophagus and stent with no leakage. $\mathbf{b}$ Esophagogram via drainage tube showing a decreased abscess cavity during follow up. c-d At 1.6 months after three-tube treatment and stent placement, a chest CT scan shows decrease in mediastinal abscess and pleural effusion 

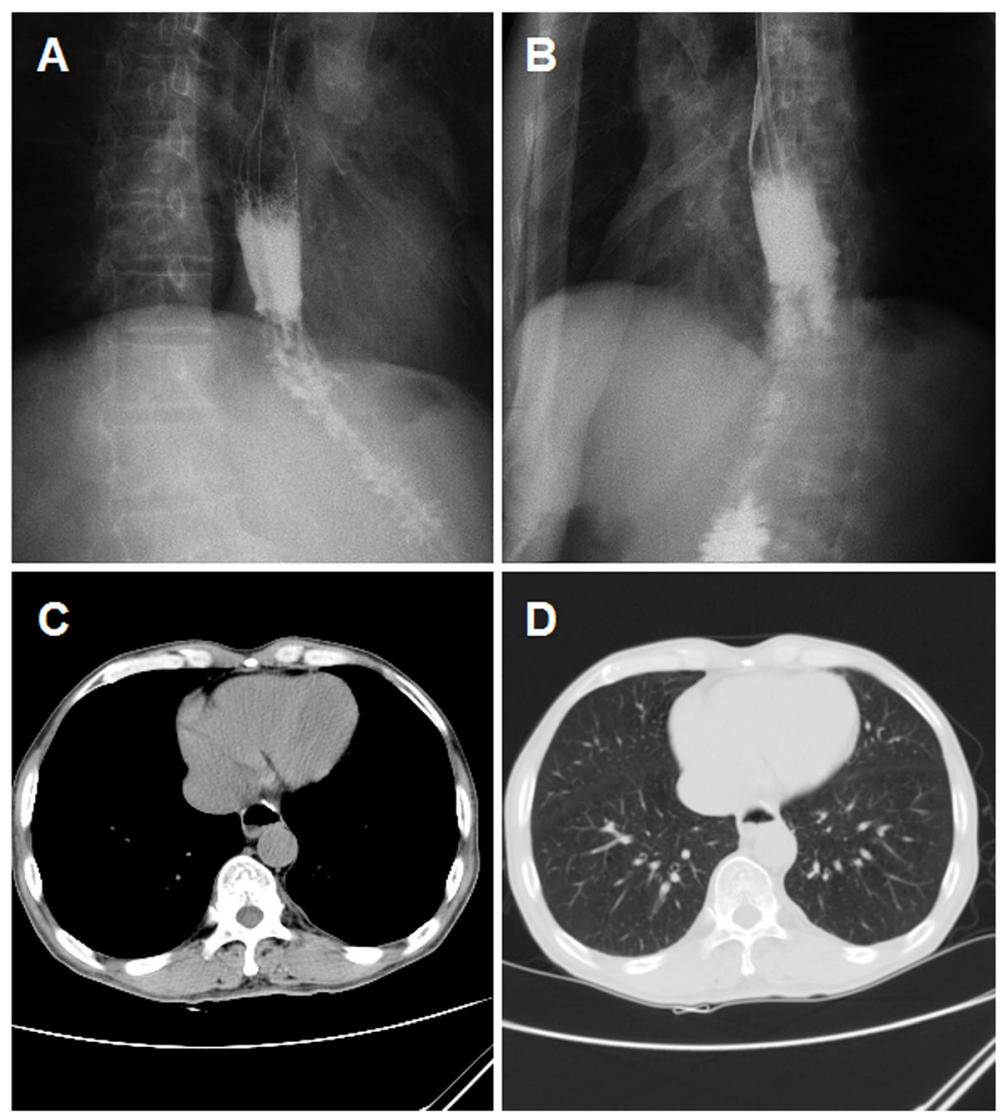

Fig. 3 Examination after stent removal for case 24. a-b The drainage tube and stent were removed after procedure; esophagography shows that the contrast agent flows though the esophagus without any leakage. c- $\mathbf{d}$ The chest $\mathrm{CT}$ scan shows disappearance of mediastinal abscess, full expansion of the lungs with no pleural effusion

$38.6^{\circ} \mathrm{C}$ (range: 37.5 to $40.0^{\circ} \mathrm{C}$ ). The median disease course before referral to our department was 3.0 months (range: 0.3 to 12 months). The median interval between esophageal surgery and leakage was 0.4 months (range: 0.2-9.0 months). There were 16 cases of gastroesophageal anastomotic fistula and 8 patients showed anastomotic leaks after esophagojejunostomy.

\section{Interventional procedure outcomes}

Three-tube method was performed successfully for all patients (100\%), only 1 patient failed Y stent placement due to complete occlusion (95.8\%). A total of 31 esophageal covered stents were placed, with a median diameter of $20 \mathrm{~mm}$ (range: $18-22 \mathrm{~mm}$ ), median length of $120 \mathrm{~mm}$ (range: $70-160 \mathrm{~mm}$ ). Stent placement was successful in the remaining 23 patients, with satisfactory expansion and appropriate position. For those patients, all anastomotic leakages were completely blocked and all stents fit watertight after covered stent placement confirmed by immediate post procedural esophagography. Three patients were in need of external thoracic drainage. All patients showed a reducing amount of drainage fluid, approximately 20 to $250 \mathrm{~mL}$ per day. Body temperature returned to normal within 2 weeks after the interventional treatment for patients with fever.

\section{Complications}

No perioperative death, esophageal rupture, massive hemorrhage, or other severe complications were observed during procedures. No intensive care was needed due to a worsening condition after the procedure. No necrosis due to negative pressure effects on the esophageal wall was observed after stent placement. Stent migration, the most common complication, was found in 9 patients, with a migration rate of $39.1 \%(9 / 23)$. Three patients showed stent restenosis, with a restenosis rate of $13.0 \%(3 / 23)$. All migrated or restenosed stents were adjusted or replaced for 1 to 3 times (median: 1.0 time). Migration of abscess drainage tube was found in 1 patient. The abscess drainage tube was adjusted or replaced for 0 to 6 times (median: 2 times). 


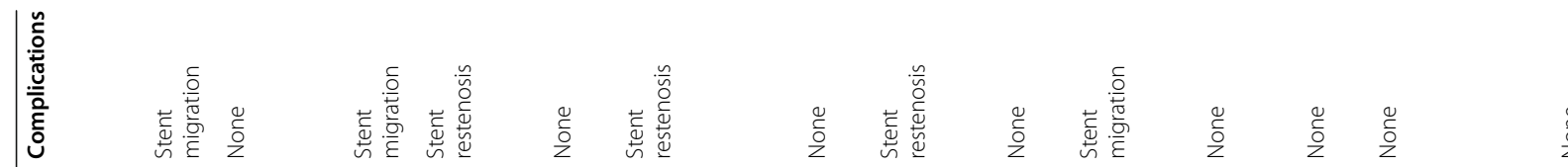

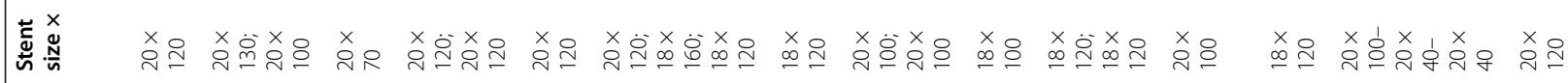

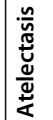

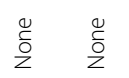

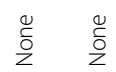

$\stackrel{\square}{\frac{0}{0}} \stackrel{0}{\frac{0}{2}}$

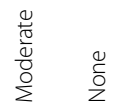

$\stackrel{\frac{0}{5}}{2}$

$\frac{\pi}{2}$

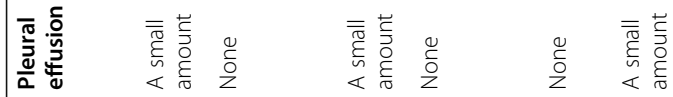

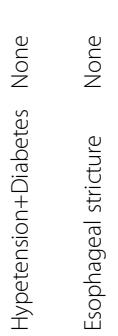

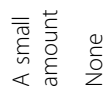

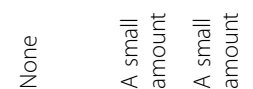

$\frac{\xi}{\frac{\xi}{\bar{m}}}$

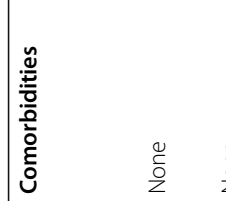

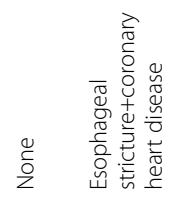

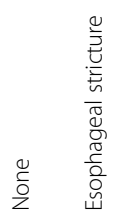

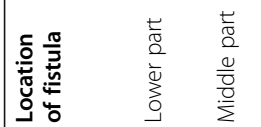

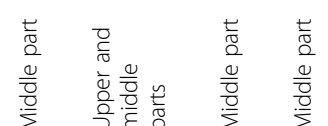

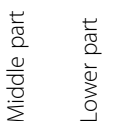

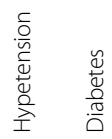

$\stackrel{8}{2}$

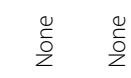

农

产

产

ลู นู

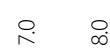

$\underset{\infty}{+} \quad \bar{N}$

นู $\stackrel{\infty}{+\infty}$

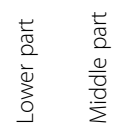

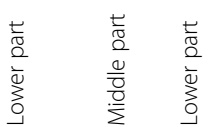

蒿

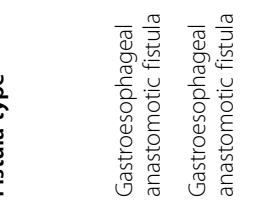

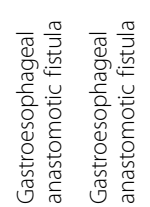

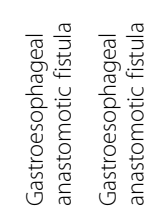

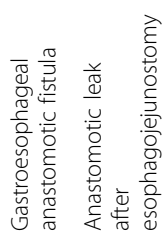

$\stackrel{m}{\sim} \stackrel{\infty}{-}$

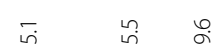

$\bar{s}$

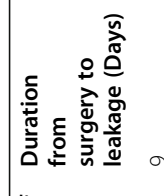

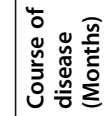

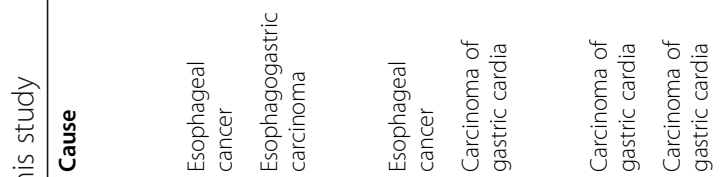

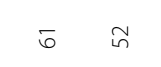

$\stackrel{\infty}{\circ}$

in

잉
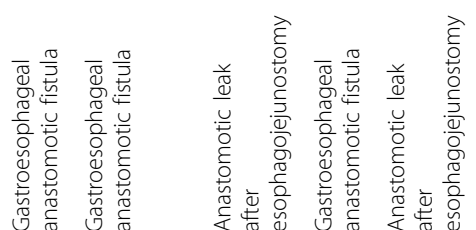

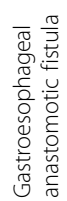




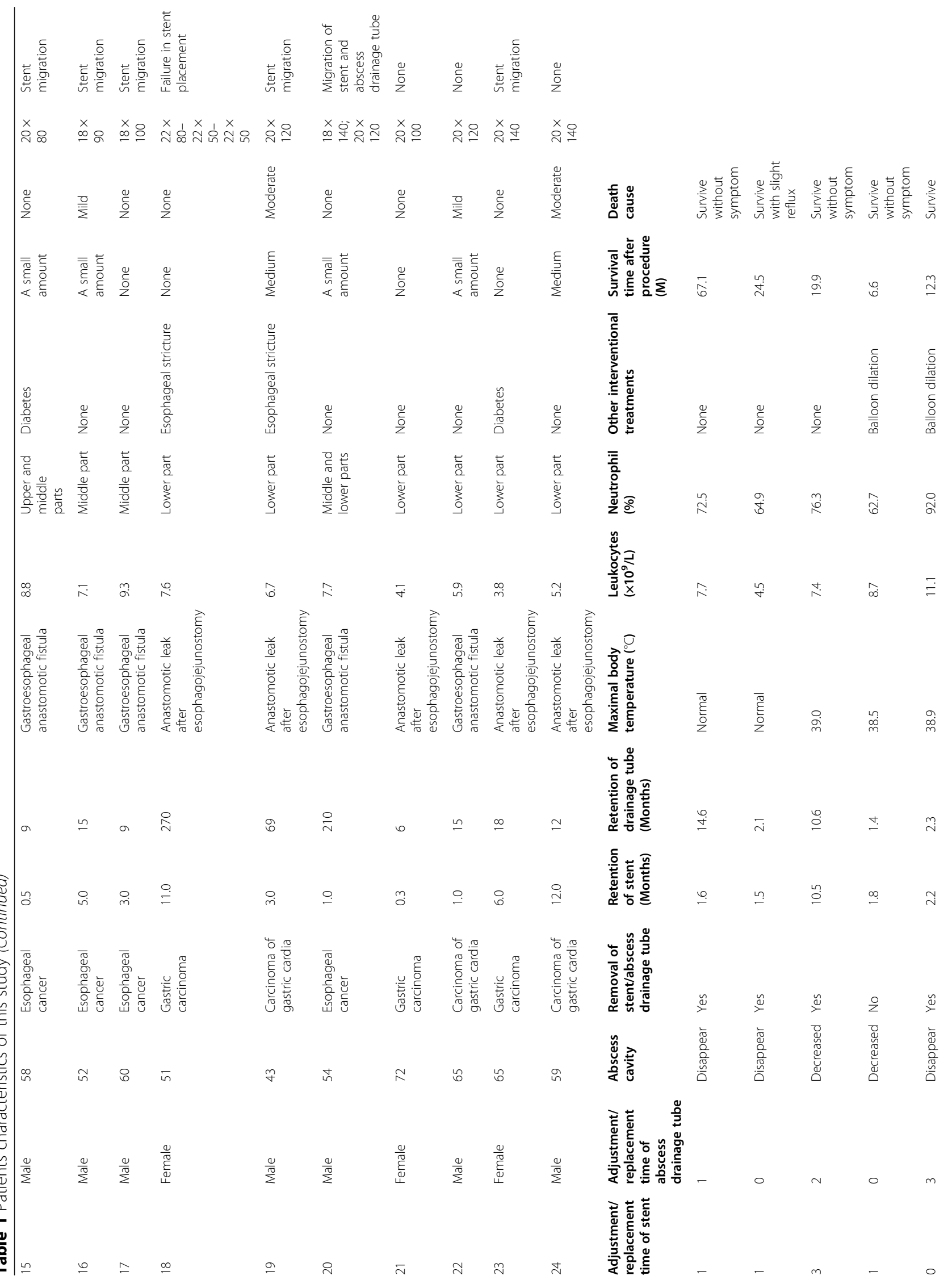




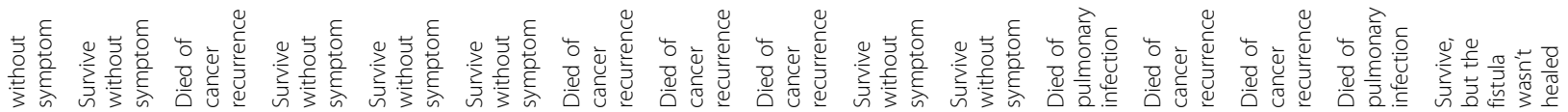

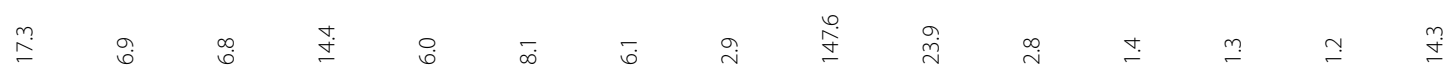

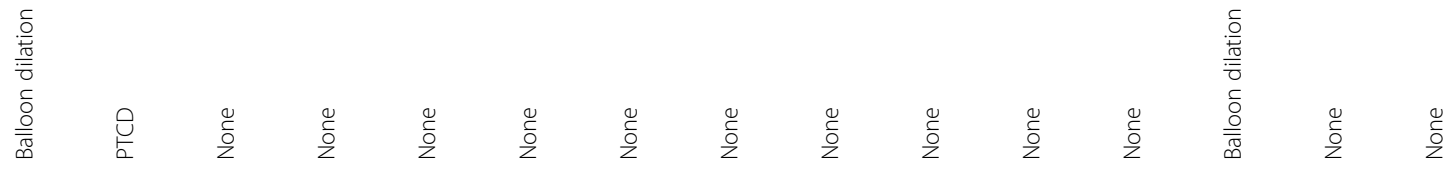

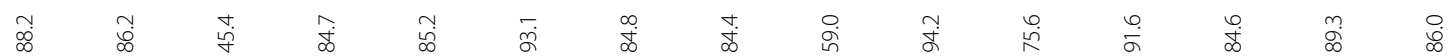

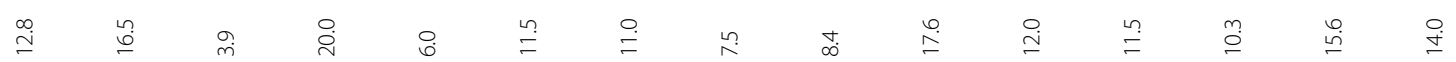

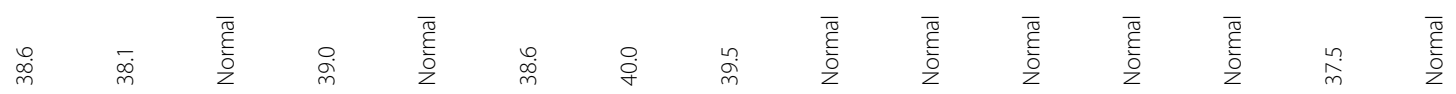

in

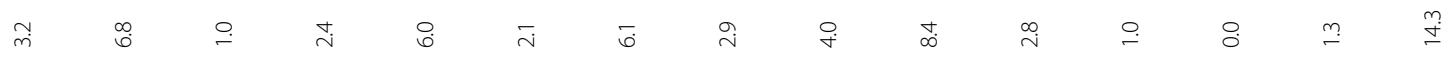

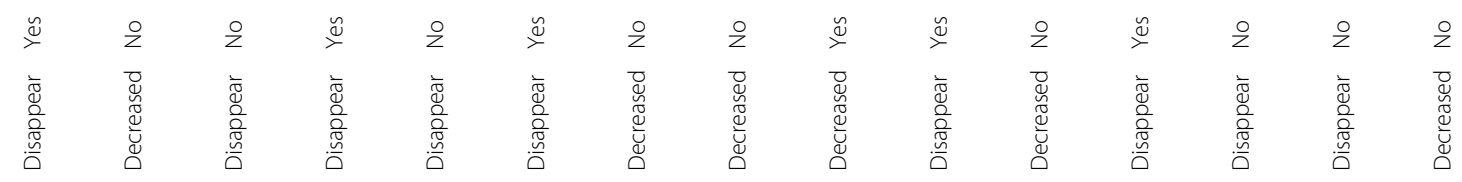




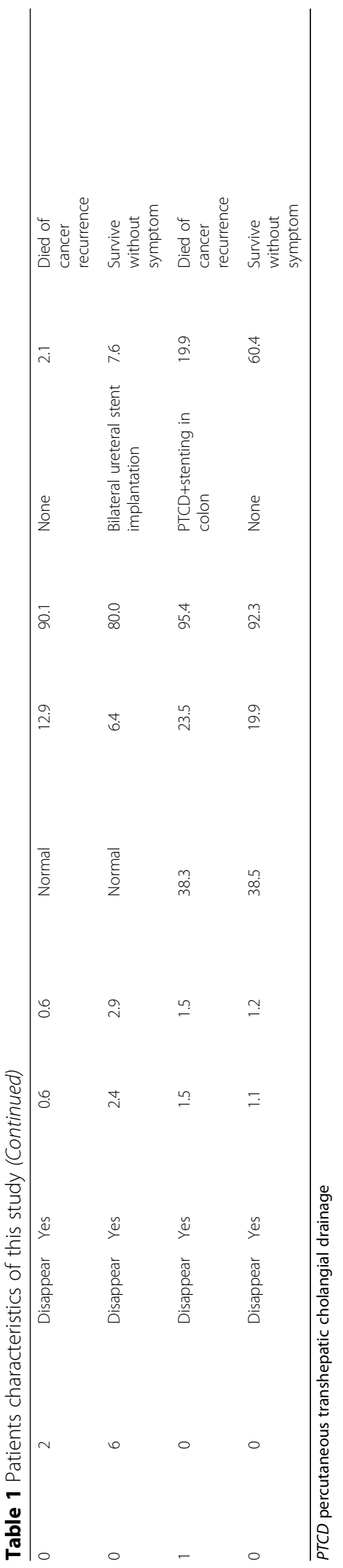




\section{Follow-up}

All patients were successfully followed up, with a median time of 7.9 months (range: $1.2-147.6$ months). Chest CT and esophagography showed that the abscess cavity had markedly decreased in 8 patients or disappeared in 16 cases. A higher rate of abscess cavity resolution was found in smaller leaks compared to that of large leaks $(6 / 12)$. However, this different was not statistically significant $(P=0.11)$, due to small sample size. During follow up, esophageal stents and abscess drainage tubes were successfully removed from 14 patients. The remaining patients refused to remove stent due to heavy financial burden and fear of possible risks of removal. The median retention duration was 2.3 months (range, $0-14.3$ months) for stents and 2.6 months (range, 0.418.6 months) for abscess drainage tubes, respectively. To this date 14 patients are still alive, with 12 patients returning to their normal daily activities of living and symptom free and 1 patient with slight reflux which is not requiring any treatment. During follow up, 8 patients died of cancer recurrence and 2 patients died of severe pulmonary infection. The 1-, 3-, 5-year survival rates were 60.1, 51.5 and 51.5\%, respectively (Fig. 4). A higher rate of 1-year survival rate $(70.7 \%)$ was found in small leaks than that of large leaks (48.6\%). However, this different was not statistically significant $(P=0.31)$.

\section{Discussion}

Management of anastomotic leakage is challenging for patients received esophagectomy The mortality is high, however, the optimal treatment still need to be determined $[2,4,11]$. Various conservative treatment protocols have been reported for the treatment of anastomotic leakage over the past two decades, including the application of biodegradable fistulae plugs or fibrin glue, endoscopic transluminal drainage or clipping and metallic esophageal stent insertion [4, 8-10]. Surgical repair of the anastomotic leakage is the traditional protocol, such as, esophagectomy or thoracotomy and repair [12]. Despite of the advances in surgical technique, the overall mortality remains as high as 20 to $50 \%$ [3-6]. Nowadays, treatment of choice in the first line is an endoscopic approach.

Esophageal stents were initially served as a palliative treatment for patients with esophageal carcinoma. Currently, esophageal stents have been used to treat esophageal benign diseases [13-17]. Metallic stents are usually used for treatment of tumor stenosis or esophageal perforation $[18,19]$. Successful and effective management of anastomotic leakage needs prompt elimination of contamination by covered stent placement, and adequate drainage of the abscess cavity. We present 24 consecutive patients treated with three-tube method and covered stent placement for anastomotic leakage. Our clinical outcomes indicated that this interventional method can easily be performed under fluoroscopic guidance. No perioperative death was observed, which is lower than previous reports $[6,15,16,20]$. After covered stent placement, the leakage is still allowed to continuously drain fluid via abscess drainage tube. In our study, all patients received continue abscess drain for a median duration of 2.6 months. Drainage of an abscess cavity is also possible percutaneously under CT scan control and it is generally easier for the subsequent follow-up. In this study, three patients were in need of external thoracic drainage. Compared with endoscopic drainage, radiologic drainage can be performed via the leaks without the need of percutaneous puncture.

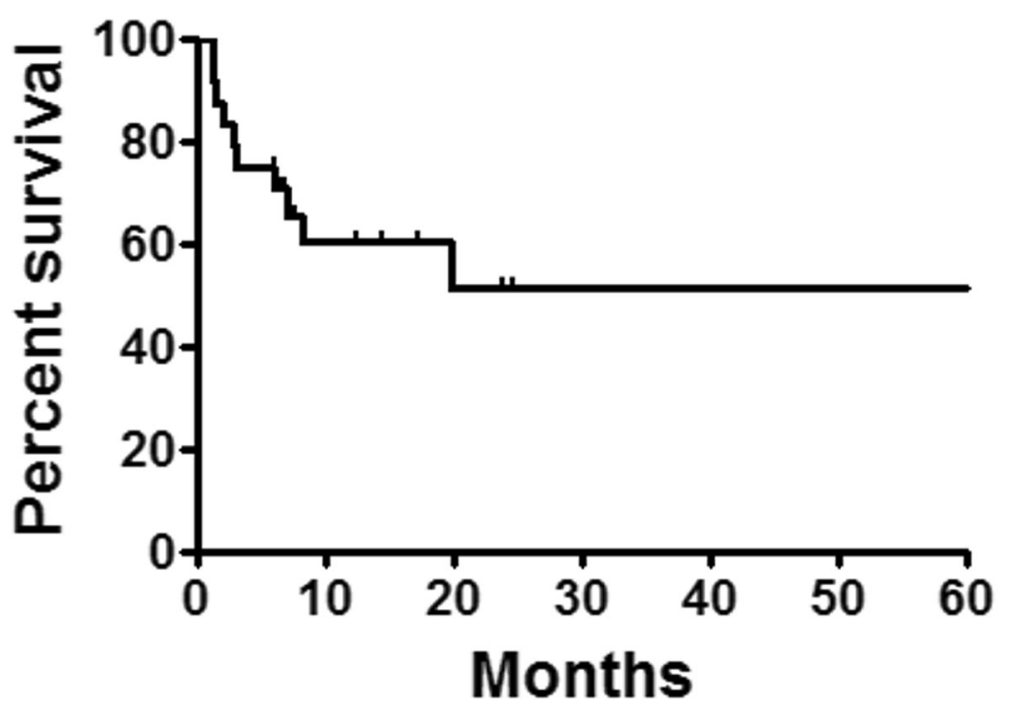

Fig. 4 Survivals follow up. The 1-, 3-, 5-year survival rates were 60.1, 51.5 and 51.5\%, respectively 
The duration between esophageal surgery and leakage diagnosis is essential for the clinical outcomes [12]. The median interval between surgery and leakage was 0.4 months. The clinical outcomes were favorable; abscess cavity was markedly decreased in 8 patients and disappeared in 16 cases. Treatment results may be related to the size of the leak, and effective results of biodegradable fistulae plugs or fibrin glue application are generally observed only in small leaks.

Certain complications can be found in our interventional protocol. Stent migration is a common complication, especially in patients without esophageal stricture $[21,22]$. All esophageal stents used in our study were coved ones, which may account for high rate of stent migration. Nine patients showed stent migration and were adjusted or replaced for 1 to 3 times. Only 1 patient showed migration of drainage tube, however, drainage tubes were regularly adjusted and replaced for 0 to 6 times to achieve effective drainage during follow up. The abscess drainage tubes were adjusted or exchanged for a median time of 2 times. Besides, esophageal stents and abscess drainage tubes were successfully removed from 14 patients, without difficulties of removal or severe complications. Recovery lines in the proximal end of stent are used for stent fixation to avoid stent migration, and for the adjustment or recovery of the migrated stent. Appropriate size of stent should been used, considering that small size of stent is prone to migrate. Of course, clips and stent with flaps can be used to reduce the migration rate.

There were some limitations. This was a retrospective study with relatively small number of enrolled patients. The esophageal stents and abscess drainage tubes needed adjusted or replaced repeatedly during follow up. BMI data had not collected previously considering that BMI may be not closely related to our treatment. We had not measured how much negative pressure and the study interval was long.

\section{Conclusions}

Three-tube method and covered stent placement can be considered a safe and effective alternative to operative treatment for anastomotic leakage after esophagectomy. Combined interventional protocol with additional supportive therapy is useful to achieve good clinical outcomes.

\section{Abbreviations}

$\mathrm{CT}$ : Computerized tomography; IQR: Mean \pm interquartile range

\section{Acknowledgements}

None

\section{Authors' contributions}

Concept and design: XH and JR; Data collection: ZW; Manuscript drafting: ZW, YB and MY; Statistical analysis: YB and MY; Administrative support: XH and JR; all authors discussed the results and revised the manuscript. The author(s) read and approved the final manuscript.

\section{Funding}

This work was supported by the National Natural Science Foundation of China (Grant No. 81501569). The funders had no role in study design, data collection and analysis, decision to publish or preparation of the manuscript.

Availability of data and materials

For further details, the corresponding author can be contacted.

\section{Ethics approval and consent to participate}

This study was approved by the Ethics Committee of The First Affiliated Hospital of Zhengzhou University. Written informed consents were obtained from all patients.

\section{Consent for publication}

Written informed consents were obtained from the patients for publication. A copy of the written consent is available for review by the Editor of this journal.

\section{Competing interests}

None of the authors has any conflict of interest to disclose.

Received: 14 July 2020 Accepted: 29 September 2020

Published online: 07 October 2020

\section{References}

1. Safranek PM, Cubitt J, Booth MI, Dehn TC. Review of open and minimal access approaches to oesophagectomy for cancer. Br J Surg. 2010;97(12): 1845-53.

2. Pennathur A, Luketich JD. Resection for esophageal cancer: strategies for optimal management. Ann Thorac Surg. 2008;85(2):S751-6.

3. Junemann-Ramirez M, Awan MY, Khan ZM, Rahamim JS. Anastomotic leakage post-esophagogastrectomy for esophageal carcinoma: retrospective analysis of predictive factors, management and influence on longterm survival in a high volume centre. Eur J Cardiothorac Surg. 2005;27(1):3-7.

4. Zheng YZ, Dai SQ, Shan HB, Gao XY, Zhang LJ, Cao X, et al. Managing esophageal fistulae by endoscopic transluminal drainage in esophageal cancer patients with superior mediastinal sepsis after esophagectomy. Chin J Cancer. 2013;32(8):469-73.

5. Blewett CJ, Miller JD, Young JE, Bennett WF, Urschel JD. Anastomotic leaks after esophagectomy for esophageal cancer: a comparison of thoracic and cervical anastomoses. Ann Thorac Cardiovasc Surg. 2001;7(2):75-8.

6. Hofstetter W, Swisher SG, Correa AM, Hess K, Putnam JB Jr, Ajani JA, et al. Treatment outcomes of resected esophageal cancer. Ann Surg. 2002;236(3): 376-84 discussion 84-5.

7. Urschel JD. Esophagogastrostomy anastomotic leaks complicating esophagectomy: a review. Am J Surg. 1995;169(6):634-40.

8. Toussaint E, Eisendrath P, Kwan V, Dugardeyn S, Deviere J, Le Moine O. Endoscopic treatment of postoperative enterocutaneous fistulas after bariatric surgery with the use of a fistula plug: report of five cases. Endoscopy. 2009;41(6):560-3.

9. Leers JM, Vivaldi C, Schafer H, Bludau M, Brabender J, Lurje G, et al. Endoscopic therapy for esophageal perforation or anastomotic leak with a self-expandable metallic stent. Surg Endosc. 2009;23(10):2258-62.

10. Lippert E, Klebl FH, Schweller F, Ott C, Gelbmann CM, Scholmerich J, et al. Fibrin glue in the endoscopic treatment of fistulae and anastomotic leakages of the gastrointestinal tract. Int J Colorectal Dis. 2011;26(3):303-11.

11. Lang H, Piso P, Stukenborg C, Raab R, Jahne J. Management and results of proximal anastomotic leaks in a series of 1114 total gastrectomies for gastric carcinoma. Eur J Surg Oncol. 2000;26(2):168-71.

12. Brinster CJ, Singhal S, Lee L, Marshall MB, Kaiser LR, Kucharczuk JC. Evolving options in the management of esophageal perforation. Ann Thorac Surg. 2004;77(4):1475-83.

13. Fischer A, Thomusch O, Benz S, von Dobschuetz E, Baier P, Hopt UT. Nonoperative treatment of 15 benign esophageal perforations with selfexpandable covered metal stents. Ann Thorac Surg. 2006;81(2):467-72.

14. Peters JH, Craanen ME, van der Peet DL, Cuesta MA, Mulder CJ. Selfexpanding metal stents for the treatment of intrathoracic esophageal 
anastomotic leaks following esophagectomy. Am J Gastroenterol. 2006; 101(6):1393-5.

15. Freeman RK, Van Woerkom JM, Ascioti AJ. Esophageal stent placement for the treatment of iatrogenic intrathoracic esophageal perforation. Ann Thorac Surg. 2007;83(6):2003-7 discussion 7-8.

16. Hunerbein M, Stroszczynski C, Moesta KT, Schlag PM. Treatment of thoracic anastomotic leaks after esophagectomy with self-expanding plastic stents. Ann Surg. 2004;240(5):801-7.

17. Kauer WK, Stein HJ, Dittler HJ, Siewert JR. Stent implantation as a treatment option in patients with thoracic anastomotic leaks after esophagectomy. Surg Endosc. 2008;22(1):50-3.

18. Siersema PD. Treatment of esophageal perforations and anastomotic leaks: the endoscopist is stepping into the arena. Gastrointest Endosc. 2005;61(7): 897-900.

19. White RE, Mungatana C, Topazian M. Expandable stents for iatrogenic perforation of esophageal malignancies. J Gastrointest Surg. 2003;7(6):715-9 discussion 9-20.

20. Freeman RK, Ascioti AJ, Wozniak TC. Postoperative esophageal leak management with the Polyflex esophageal stent. J Thorac Cardiovasc Surg. 2007;133(2):333-8

21. Schubert D, Scheidbach H, Kuhn R, Wex C, Weiss G, Eder F, et al. Endoscopic treatment of thoracic esophageal anastomotic leaks by using silicone-covered, self-expanding polyester stents. Gastrointest Endosc. 2005; 61(7):891-6.

22. Gelbmann CM, Ratiu NL, Rath HC, Rogler G, Lock G, Scholmerich J, et al. Use of self-expandable plastic stents for the treatment of esophageal perforations and symptomatic anastomotic leaks. Endoscopy. 2004;36(8): 695-9.

\section{Publisher's Note}

Springer Nature remains neutral with regard to jurisdictional claims in published maps and institutional affiliations.

Ready to submit your research? Choose BMC and benefit from:

- fast, convenient online submission

- thorough peer review by experienced researchers in your field

- rapid publication on acceptance

- support for research data, including large and complex data types

- gold Open Access which fosters wider collaboration and increased citations

- maximum visibility for your research: over $100 \mathrm{M}$ website views per year

At $\mathrm{BMC}$, research is always in progress.

Learn more biomedcentral.com/submissions 